\title{
New York City COVID-19 resident physician exposure during exponential phase of pandemic
}

\author{
Mark P. Breazzano, ${ }^{1,2,3}$ Junchao Shen, ${ }^{4}$ Aliaa H. Abdelhakim, ${ }^{1,2,3}$ Lora R. Dagi Glass, ${ }^{1}$ Jason D. Horowitz, ${ }^{1}$ Sharon X. Xie, ${ }^{5}$ \\ C. Gustavo de Moraes, ${ }^{1}$ Alice Chen-Plotkin, ${ }^{4}$ andRoyce W.S. Chen. ${ }^{1}$, on behalf of the New York City Residency Program Directors \\ COVID-19 Research Group ${ }^{6}$

\begin{abstract}
'Department of Ophthalmology, Edward S. Harkness Eye Institute, Columbia University Irving Medical Center, New York-Presbyterian Hospital, New York, New York, USA. ²Department of Ophthalmology, New York University School of Medicine, New York University Langone Health, New York, New York, USA. ${ }^{3}$ Manhattan Eye, Ear and Throat Hospital, Lenox Hill Hospital, Northwell Health, New York, New York, USA. ${ }^{4}$ Department of Neurology and ${ }^{5}$ Department of Biostatistics, Epidemiology and Informatics, Perelman School of Medicine, University of Pennsylvania, Philadelphia, Pennsylvania, USA. ${ }^{6}$ The New York City Residency Program Directors COVID-19 Research Group is detailed in the Supplemental Acknowledgments.
\end{abstract}

BACKCROUND. From March 2, 2020, to April 12, 2020, New York City (NYC) experienced exponential growth of the COVID-19 pandemic due to novel coronavirus (SARS-CoV-2). Little is known regarding how physicians have been affected. We aimed to characterize the COVID-19 impact on NYC resident physicians.

METHODS. IRB-exempt and expedited cross-sectional analysis through survey to NYC residency program directors April 3-12, 2020, encompassing events from March 2, 2020, to April 12, 2020.

RESULTS. From an estimated 340 residency programs around NYC, recruitment yielded 91 responses, representing 24 specialties and 2306 residents. In $45.1 \%$ of programs, at least 1 resident with confirmed COVID-19 was reported. One hundred one resident physicians were confirmed COVID-19-positive, with an additional 163 residents presumed positive for COVID-19 based on symptoms but awaiting or unable to obtain testing. Two COVID-19-positive residents were hospitalized, with 1 in intensive care. Among specialties with more than 100 residents represented, negative binomial regression indicated that infection risk differed by specialty $(P=\mathbf{0 . 0 3 9})$. In $80 \%$ of programs, quarantining a resident was reported. Ninety of 91 programs reported reuse or extended mask use, and 43 programs reported that personal protective equipment (PPE) was suboptimal. Sixty-five programs (74.7\%) redeployed residents elsewhere to support COVID-19 efforts.

CONCLUSION. Many resident physicians around NYC have been affected by COVID-19 through direct infection, quarantine, or redeployment. Lack of access to testing and concern regarding suboptimal PPE are common among residency programs. Infection risk may differ by specialty.

FUNDING. National Eye Institute Core Grant P30EY019007; Research to Prevent Blindness Unrestricted Grant; Parker Family Chair; University of Pennsylvania.

\section{Introduction}

The United States is part of a global pandemic known as COVID-19 (1), which has characteristics that overlap with the Spanish flu of 1918. The causative novel coronavirus (2019-nCoV, SARS-CoV-2), first described in Wuhan, China $(2,3)$, has spread worldwide, particularly in New York City (NYC), which was recently the US epicenter of cases and mortality (4). The first case was confirmed in NYC on March 1, 2020 (5); 6 weeks later, hundreds of patients were dying from COVID-19 daily (6). Healthcare workers are on the front lines of this pandemic $(2,7)$. However, although at least 4500

Related Commentary: p. 4543

Conflict of interest: The authors have declared that no conflict of interest exists. Copyright: () 2020, American Society for Clinical Investigation.

Submitted: April 27, 2020; Accepted: May 21, 2020; Published: August 4, 2020.

Reference information: / Clin Invest. 2020;130(9):4726-4733.

https://doi.org/10.1172/JCl139587. peer-reviewed articles have been published on this topic between January 1, 2020, and April 18, 2020, comparatively little is known about the toll of COVID-19 on health care workers directly occupied with patient care.

Notably, the first physician to sound the alarm about the novel coronavirus causing severe acute respiratory syndrome coronavirus 2 (SARS-CoV-2) was the Chinese ophthalmologist Li Wenliang, who died after contracting the infection from a presymptomatic patient (8). Anecdotally, health care workers in NYC have experienced unique challenges in combatting the illness, including close contact with the sickest patients, exposure to high viral loads, redeployment to clinical duties outside of their ordinary responsibilities, and severe shortages in personal protective equipment (PPE) $(7,9,10)$. Among those at highest risk are resident physicians, who are commonly stationed in high-acuity settings and comprise a substantial part of the health care workforce in the United States (11). The activities of resident physicians are standardized among residency training programs throughout the 
United States via accreditation with the Accreditation Council for Graduate Medical Education (ACGME), with each residency program supervised by an appointed program director (12). The structure of residency programs, in which many resident physicians report to 1 program director responsible for their activities and well-being, makes the resident physician population practical for study through collection of data from residency program directors. However, to our knowledge, no primary peer-reviewed data have addressed implications of COVID-19 for resident physicians, whose situation has only been described in editorials $(13,14)$. We also sought to explore whether specialty-specific risks existed for COVID-19 infection. By surveying residency program directors across all specialties in NYC from April 3-12, 2020, we captured the immediate features and impact of COVID-19 among resident physicians during the exponential phase of the COVID-19 pandemic in NYC. Because future or recurrent outbreaks are likely, such knowledge may help tailor future interventions to mitigate the burden of COVID-19 among health care workers.

\section{Results}

Study sample. One hundred two program director responses were received between April 3-12, 2020, 10 of which were excluded because the represented programs did not satisfy residency and ACGME-accreditation criteria (i.e., they were fellowship rather than residency programs), and 1 of which was removed as it was incomplete and reported zero residents in the program. Thus, 91 programs representing 2306 residents from 24 different specialties were included in this study (Figure 1). Average program size was 25 residents $(\mathrm{SD}=21)$, with a range of $1-98$ residents per program. Forty-nine programs $(53.8 \%, 95 \%$ CI 43.1-64.4) reported that residents provided services for at least 3 different hospitals.

Overall cases and testing frequency of COVID-19. All 91 program directors reported numbers for symptomatic residents who had tested positive for COVID-19 ("confirmed" cases). Ninety of 91 program directors reported numbers for symptomatic residents who were awaiting or unable to obtain testing ("presumed" cases) and symptomatic residents who had tested negative for COVID-19 ("suspected" cases). In total, 41 of 91 (45.1\%, 95\% CI 34.6-55.8) programs reported at least 1 confirmed case, 49 of 90 programs (54.4\%, 95\% CI 43.6-65.0) reported at least 1 presumed case, and 36 of 90 programs (40\%, 95\% CI $29.8-50.9)$ reported at least 1 suspected case. Among all residents from all programs pooled together, 101 residents were confirmed cases, 163 were presumed cases, and 76 were suspected cases (Figure 2). The total number and proportion of affected residents by specialty are shown in Table 1 .

Eighty-six of 91 program directors reported knowing how many residents were tested for COVID-19. Among the 2088 residents in these 86 programs, a total of 242 residents $(11.6 \%, 95 \%$ CI 10.2-13.0) were tested for COVID-19. One hundred seventy-seven residents who were tested also had results reported by the time of the survey. Among these, 101 (57.1\%, 95\% CI 49.4-64.5) tested positive and 76 (42.9\%, 95\% CI 35.5-50.6) were negative.

Sixty-nine of 91 program directors reported knowing the exact number of residents who were tested for COVID-19 as well as whether residents were awaiting testing. Among 1673 residents in these 69 programs, 113 residents $(6.8 \%, 95 \%$ CI 5.6-8.1) were waiting for or unable to obtain testing. Thirty-nine (56.5\%, $95 \% \mathrm{CI}$
44.0-68.4) residency programs had at least 1 resident waiting for or unable to get testing.

For residents who tested positive for COVID-19 as well as those who tested negative, the majority of testing was performed with reverse transcription polymerase chain reaction (RT-PCR) of samples collected by nasal swab $(n=85$ [84.2\%] for test-positive; $n=59$ [77.6\%] for test-negative), followed by oropharyngeal swab ( $n=5[5.2 \%]$ for test-positive; $n=6$ [7.9\%] for test-negative).

Disease burden by specialty. To determine whether any specific medical specialties were more likely to have a COVID-19-positive resident, all specialties with more than 100 residents in our sample were compared. Programs that met this criterion included anesthesiology, emergency medicine, general surgery, internal medicine, ophthalmology, pediatrics, and psychiatry (Figure 1). Three specialties (anesthesiology, emergency medicine, ophthalmology) appeared to cluster as high-risk specialties by proportion of residents with confirmed COVID-19, compared with the remaining specialties $(P$ $=0.015$, Fisher's exact test). In negative binomial models adjusted for the size of the residency program and date of survey completion, specialty remained significantly associated with the number of confirmed positive residents $(P=0.039)$. Using anesthesiology as the reference group (as this specialty had the highest proportion of positive residents), anesthesiology was significantly more likely to have a COVID-19-confirmed resident, compared with both internal medicine $(P=0.020)$ and pediatrics $(P=0.029)$.

Timing of symptom onset. Symptom onset was reported to occur as early as or before the week of March 2-8, 2020, for 5 residents $(1.5 \%)$ with confirmed $(n=1)$, presumed $(n=3)$, or suspected $(n=$ 1) COVID-19 (Figure 3). Most residents with confirmed COVID-19 ( $n=35,34.7 \%, 95 \%$ CI 25.5-44.8) were reported to first experience symptoms the week of March 22-28, 2020. By contrast, most with presumed ( $n=53,32.5 \%, 95 \%$ CI 25.4-40.3) and suspected ( $n=29,38.2 \%, 95 \%$ CI 27.2-50.0) COVID-19 reported symptoms beginning the week of March 15-21, 2020. Symptom onset for affected residents among every category (confirmed: $n=3,3.0 \%$; presumed: $n=3,1.8 \%$; suspected: $n=1,1.3 \%$ ) continued through the last week of survey participation, April 6-12, 2020.

Personal protective equipment (PPE). The majority of programs, encompassing 1832 residents (79.4\%, 95\% CI 77.7-81.1) used either N95 or surgical masks during patient encounters, depending on the context. Nineteen programs encompassing 323 residents (14\%, 95\% CI 12.6-15.5) used only surgical masks during patient encounters, and 8 programs encompassing 31 residents $(5.7 \%$, 95\% CI 4.8-6.7) used an N95 respirator for all patient encounters. Excepting 1 radiology program, all programs, encompassing 99.2\% of residents in this study, reported reuse or extended use of their masks (versus single use). Protocols mandating universal wearing of surgical masks were introduced as early as the week of March 2-8, 2020, in only 3 programs (3.5\%) and as late as March 30, 2020, to April 5, 2020, in 20 programs (23.5\%, Figure 3).

Forty-three of 87 program directors $(49.4 \%$, 95\% CI $38.5-$ 60.4) representing 1314 residents answered "yes" when asked whether their residents had had to work with suboptimal PPE. We found no correlation between the mask type used by residents (surgical, N95, or both) and perceived shortage of PPE. We found no correlation between programs that reported suboptimal PPE and number of COVID-19-positive residents. 


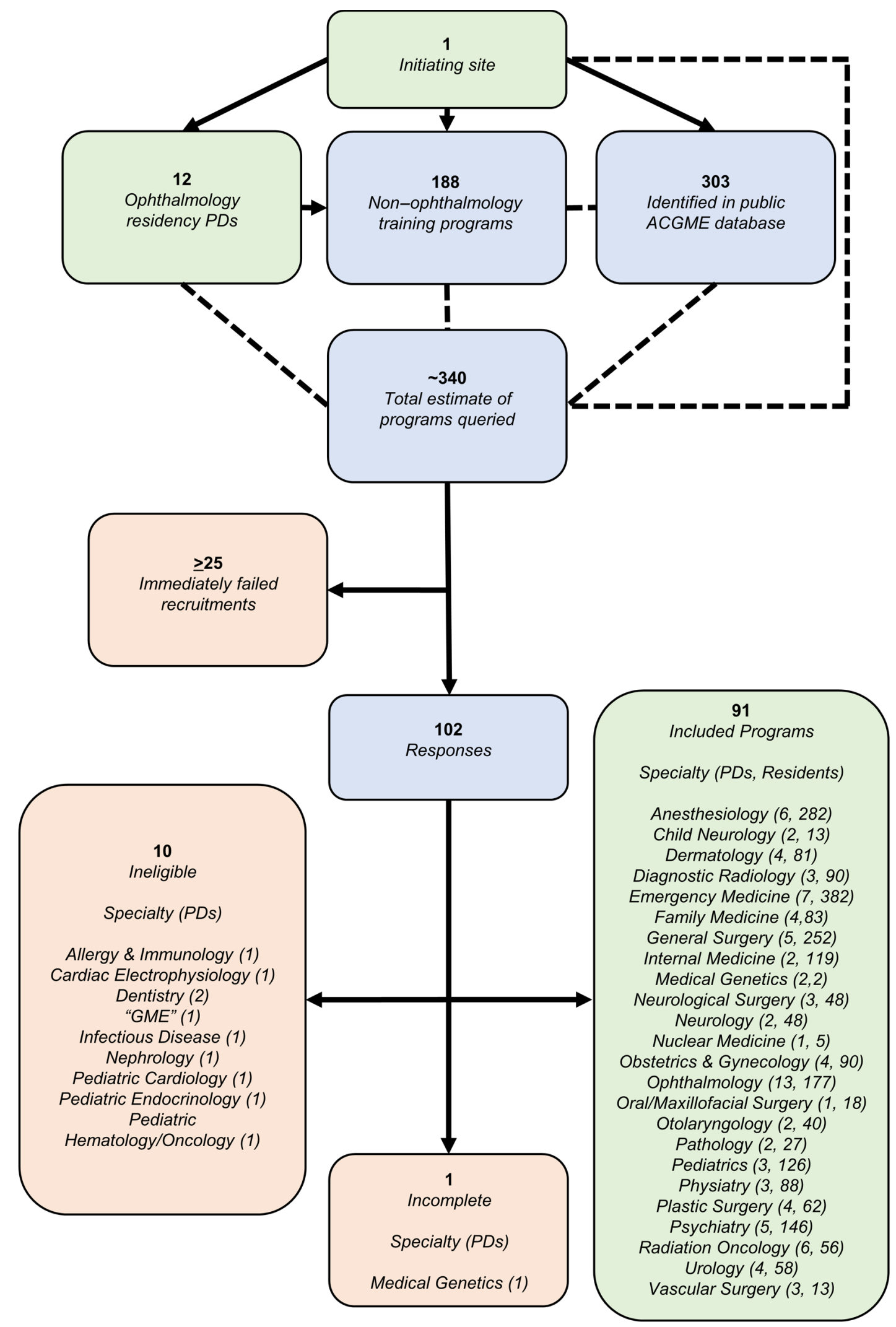

Figure 1. Flow chart of survey recruitment and responses among greater NYC training programs. The flow chart includes represented specialties and number of residents. ACGME, Accreditation Council for Graduate Medical Education; PDs, training program directors. 
Table 1. Number and percentage of symptomatic residents with confirmed (positive), presumed (untested), and suspected (negative) COVID-19 testing across specialties

\begin{tabular}{|c|c|c|c|c|}
\hline Specialty & Residents, no. & Confirmed, no. & Presumed, no. & Suspected, no. \\
\hline Vascular surgery & 13 & $4(30.8 \%)$ & $1(7.7 \%)$ & $0(0.0 \%)$ \\
\hline Emergency medicine & 382 & $25(6.5 \%)$ & $32(8.4 \%)$ & $12(3.1 \%)$ \\
\hline Ophthalmology & 177 & $9(5.1 \%)$ & $17(9.6 \%)$ & $7(4.0 \%)$ \\
\hline Otolaryngology & 40 & $2(5.0 \%)$ & $3(7.5 \%)$ & $2(5.0 \%)$ \\
\hline Plastic surgery & 62 & $3(4.8 \%)$ & $17(27.4 \%)$ & $0(0 \%)$ \\
\hline Obstetrics and gynecology & 90 & $4(4.4 \%)$ & $7(7.7 \%)$ & $1(1.1 \%)$ \\
\hline Dermatology & 81 & $3(3.7 \%)$ & $1(1.2 \%)$ & $7(9.2 \%)$ \\
\hline Pathology & 27 & $1(3.7 \%)$ & $1(3.7 \%)$ & $0(0.0 \%)$ \\
\hline General surgery & 252 & $9(3.6 \%)$ & $16(6.3 \%)$ & $13(5.2 \%)$ \\
\hline Psychiatry & 146 & $5(3.4 \%)$ & $10(6.8 \%)$ & $5(3.4 \%)$ \\
\hline Family medicine & 83 & $2(2.4 \%)$ & $3(3.6 \%)$ & $7(8.4 \%)$ \\
\hline Diagnostic radiology & 90 & $1(1.1 \%)$ & $5(5.6 \%)$ & $0(0.0 \%)$ \\
\hline Pediatrics & 126 & $1(0.7 \%)$ & $2(1.6 \%)$ & $0(0.0 \%)$ \\
\hline Urology & 58 & $0(0.0 \%)$ & $7(12.1 \%)$ & $0(0.0 \%)$ \\
\hline Child neurology & 13 & $0(0.0 \%)$ & $4(30.8 \%)$ & $1(8.6 \%)$ \\
\hline Nuclear medicine & 5 & $0(0.0 \%)$ & $1(20.0 \%)$ & $1(20.0 \%)$ \\
\hline Oral/maxillofacial surgery & 18 & $0(0.0 \%)$ & $0(0.0 \%)$ & $0(0.0 \%)$ \\
\hline Medical genetics & 2 & $0(0.0 \%)$ & $0(0.0 \%)$ & $0(0.0 \%)$ \\
\hline Total & 2306 & $101(4.4 \%)$ & $163(7.1 \%)$ & $76(3.3 \%)$ \\
\hline
\end{tabular}

Care setting and hospitalization. Among the 101 residents with confirmed COVID-19, 57 (56.4\%, 95\% CI 46.2-66.3) presented to clinic or primary care, $17(16.8 \%, 95 \%$ CI $10.1-25.6)$ visited the emergency department, 2 (2.0\%, 95\% CI 0.2-7.0) were hospitalized, and 1 (1\%, 95\% CI 0-5.4) had care escalated to the intensive care unit (ICU). The 163 residents with presumed COVID-19 presented to primary care or clinic in 40 cases $(24.5 \%, 95 \% \mathrm{CI}$ 18.1-31.9) and the emergency department in 6 cases $(3.7 \%, 95 \%$ CI 1.4-7.8). Among the 76 residents with suspected COVID-19, 38 (50\%, 95\% CI 38.3-61.7) were evaluated in clinic or by primary care, $5(6.5 \%, 95 \%$ CI $2.2-14.7)$ presented to the emergency department, and 1 (1.3\%, 95\% CI 0-7.1) was hospitalized. In total, among the 340 residents with confirmed, presumed, or suspected COVID-19, 3 (0.9\%, 95\% CI 0.2-2.6) were hospitalized (1 each from emergency medicine [who was also hospitalized and went to the ICU], ophthalmology, and psychiatry programs; 2 with confirmed and 1 with suspected COVID-19). There were no deaths reported in any of the completed surveys.

Quarantine. One program (pediatrics) of 58 residents did not report any quarantine data. Of the remaining 90 programs encompassing 2248 residents (including 339 residents with confirmed, presumed, or suspected COVID-19), 377 (16.8\%, 95\% CI $15.2-18.4$ ) residents from 72 programs (80\% of programs, $95 \%$ CI 70.2-87.7) were reported to be quarantined. Twenty-two programs (24.4\%, 95\% CI 16.0-34.6) reported at least 1 asymptomatic but exposed resident who was quarantined. Among 34 asymptomatic but exposed residents with known duration of quarantine, the time ranged from 1-14 days. Fifteen residents (14.9\%, 95\% CI 8.6-23.3) from 2 programs with confirmed COVID-19, 26 residents (16.0\%, 95\% CI 10.8-22.6) from 5 programs with presumed COVID-19, and 5 residents $(6.6 \%, 95 \%$ CI 2.2-14.7) from 2 programs with suspected COVID-19 were not quarantined.

Redeployment. Eighty-seven of 91 program directors responded to questions about residents redeployed to other departments or locations to support COVID-19 efforts. Sixty-five programs $(74.7 \%, 95 \%$ CI 64.3-83.4) reported at least 1 resident redeployed, with 35 programs $(40.2 \%$, 95\% CI 29.9-51.3) redeploying more than one-third of their workforce. Five hundred ninety-four residents (27.3\% of 2176 residents for whom redeployment information is known, 95\% CI 25.4-29.2) were reported to be redeployed. Anesthesiology had the highest redeployment rate, with 158 (56.0\% of 282 total anesthesiology residents, $95 \%$ CI 50.0-61.9) residents being redeployed to other services $(P<$ 0.001 , Pearson's $\chi^{2}$ test). Of programs that redeployed residents, 53 programs (81.5\%, 95\% CI 70.0-90.1) instituted redeployment between the fourth and fifth weeks of March, approximately 1 month after the first case in NYC was confirmed. Among residents redeployed to duties beyond their usual clinical responsibilities, the majority went to the ICU (283 of 594 redeployed residents, $47.6 \%$, 95\% CI 43.6-51.7), followed by hospital floors 


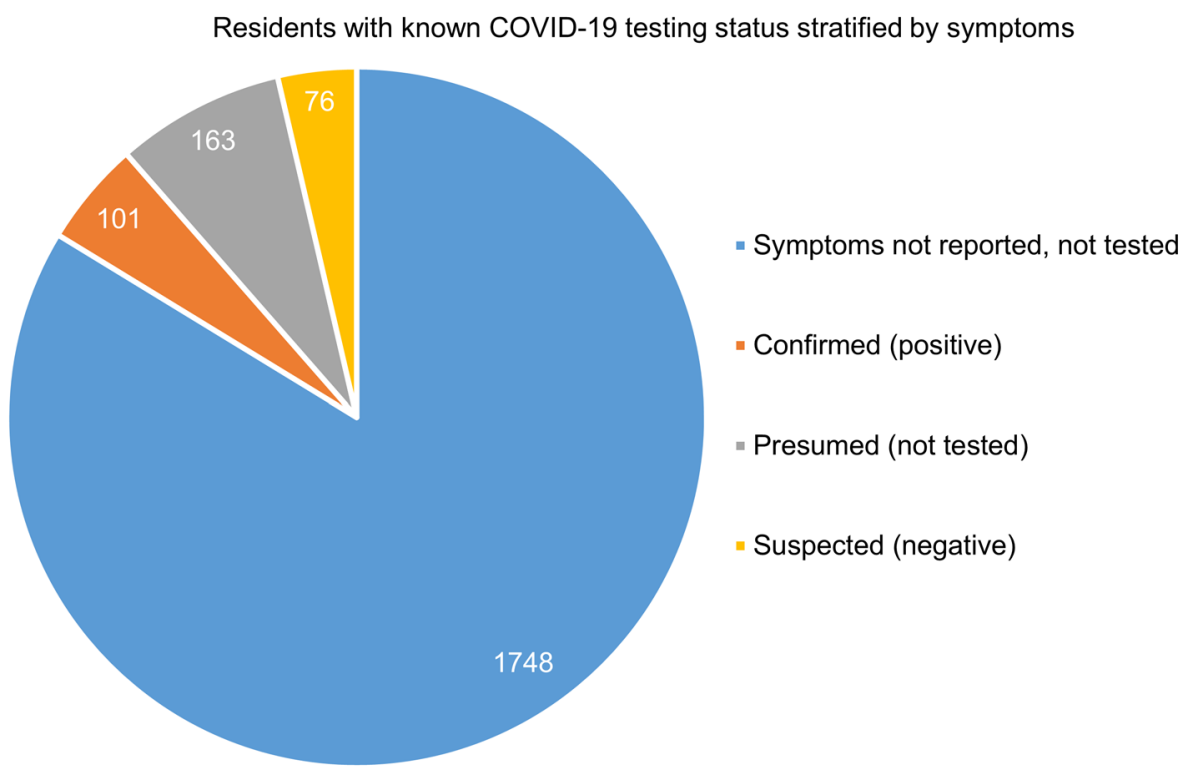

Figure 2. Residents with known COVID-19 testing status, stratified by symptoms. Of 2088 total residents with known COVID-19 testing status, 101 residents were confirmed (positive), 163 were presumed (untested), 76 were suspected (negative), and 1748 neither had symptoms nor were tested.

able after a single use. Third, we found that some specialties may be at greater risk for contracting COVID-19 compared with others. In particular, anesthesiology had significantly higher numbers of confirmed COVID-19 residents compared with internal medicine $(P=0.020)$ and pediatrics $(P=0.029)$. It is possible that the higher infection rates may be due to the critical skill of intubation provided by anesthesiologists, which comes with high probability of aerosolization and exposure to viral particles (15).

Emergency room physicians and ophthalmologists may also be at higher risk for infection. Given that emergency room physicians may intubate and are often the first-line providers for infected patients before COVID-19 status is known, it is not surprising that they segregate as a higher risk group. Factors possibly placing ophthalmologists at higher risk include close proximity to the patient's upper respiratory tract during slit lamp examination (usually less than 1

(176 of 594, 29.6\%, 95\% CI 26.0-33.5), and the emergency department ( 85 of $594,14.3 \%, 95 \%$ CI $11.6-17.4$ ).

\section{Discussion}

As of the date of our survey's close, NYC was the epicenter of the COVID-19 pandemic in the United States, and the daily death toll was continuing to rise (6). Here, we report the impact of COVID-19 on NYC resident physicians, as reported by their residency program directors, surveyed between April 3-12, 2020. Many of these residents have been directly infected (101 confirmed positive), quarantined ( $16.8 \%$ of residents), or redeployed ( $27.3 \%$ of residents) to duties outside of their usual clinical activities in support of COVID-19 efforts.

One hundred one residents were reported to have confirmed COVID-19 in our sample. While this is $4.4 \%$ of the 2306 residents whose program directors participated in our study, the true rate in our sample may be higher, since 242 resident physicians were tested for COVID-19 and only 177 had received their test results at the close of the survey.

We highlight a few points found in our study. First, program directors reported 15 confirmed COVID-19 residents and 26 presumed COVID-19 residents who were not quarantined. Whether this was due to these residents being initially asymptomatic, workforce need, delay in obtaining testing, or some other reason is not known. However, we do note that $56.5 \%$ of residency program directors reported at least 1 resident awaiting or unable to obtain COVID-19 testing. Second, $49.4 \%$ of residency directors answered "yes" to the question of whether resident physicians for whom they were responsible had suboptimal PPE. While this might reflect selection bias with respect to which residency directors chose to answer the survey, we note that 90 of 91 programs reported reuse or extended use of masks that are ordinarily dispos- ft) (16), contact with ocular secretions $(16,17)$, and high volume of patients seen in clinics.

We recognize limitations to our current study. While not all presumed and suspected cases have COVID-19, we present these numbers given the high pretest probability of infection in health care workers with suggestive symptoms, as well as known limitations of RT-PCR detection of the virus $(18,19)$. Future work using serological testing may provide a more accurate census of confirmed positives, as recent studies have shown (20), but given the limited availability of serological testing and the time-sensitive nature of our survey, this modality was not suitable for the current study. Second, we were unable to determine a relationship between mask type and proportion of COVID-19 infections for the following reasons. During the period of the study, national and local guidelines on PPE usage were continually changing based on availability and increased understanding regarding disease transmission. In addition, the majority of programs reported using both types of masks, depending on clinical context. Third, selection bias may have affected our findings, as fields such as ophthalmology may have been overrepresented due to the authors' connections to colleagues in the field, while other specialties, such as internal medicine, may have been underrepresented because of significant stress in managing overflowing COVID-19 wards and lack of time to complete the survey. It is also possible that program directors whose residents have been affected by COVID-19 would be more likely to respond. Therefore, rates of infection per specialty may need to be interpreted with caution.

However, we capture 91 NYC residency programs (out of an estimated total 340 residency programs) during a period of exponential pandemic growth, offering a unique perspective on the impact on resident physicians during what may be the height of 
Number of residents with new COVID-19 symptoms by week
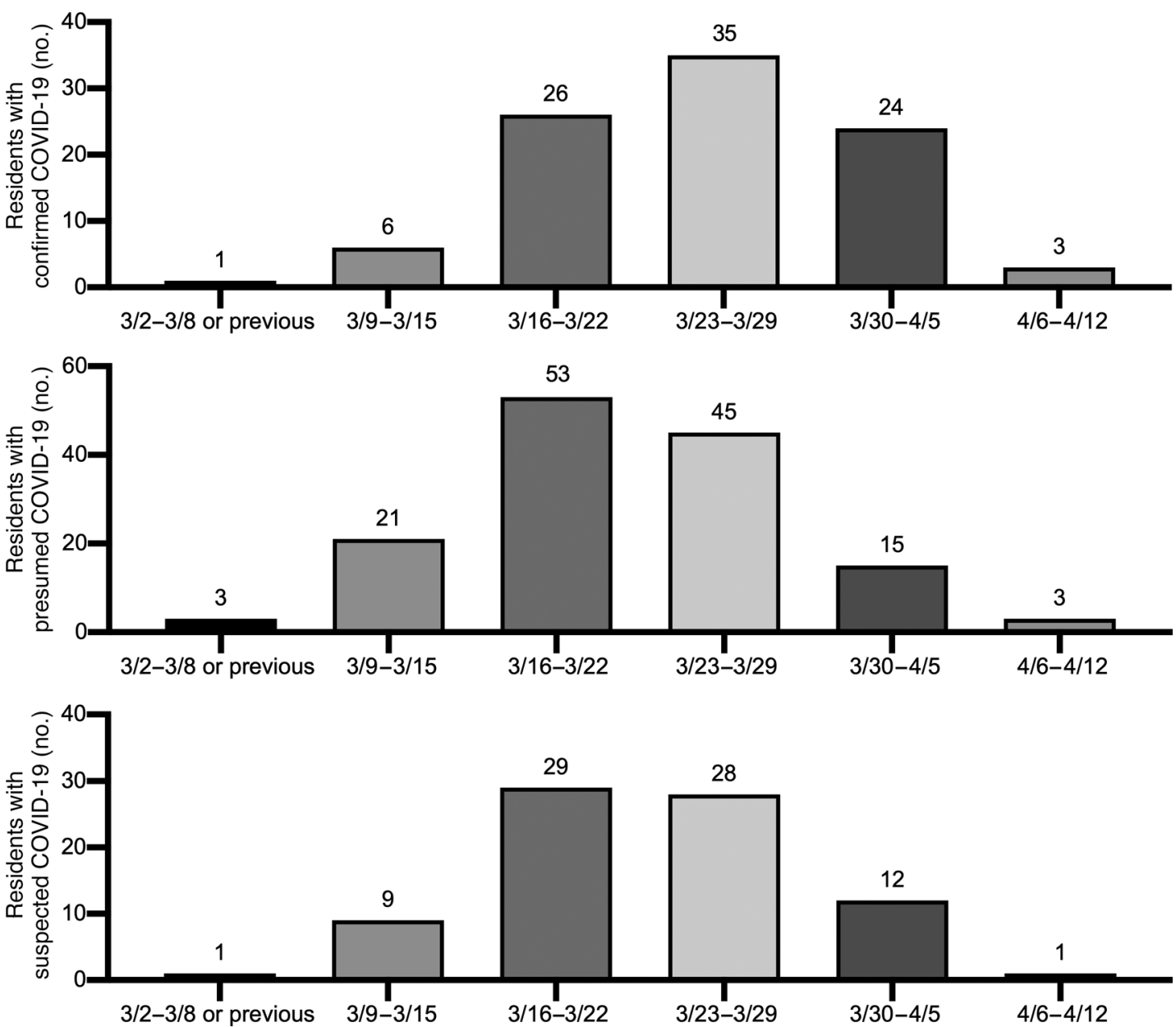

Number of residency programs enforcing universal mask policy by week

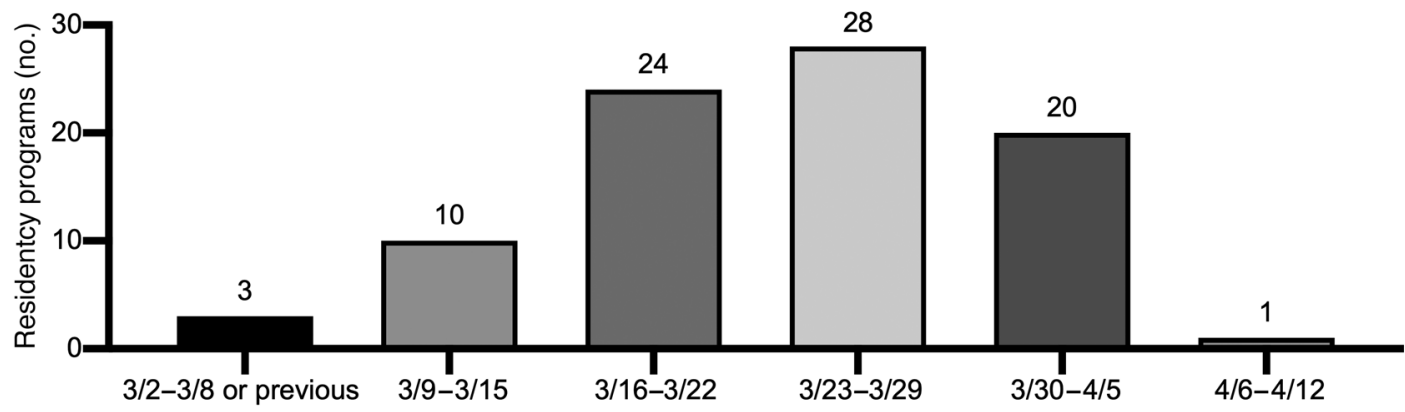

Time (wk)

Figure 3. Number of residents with new COVID-19 symptoms and number of programs enforcing mask policy, by week. Most confirmed COVID-19 cases $(n=35)$ were reported during the week of 3/23-3/29. Most presumed COVID-19 cases $(n=53)$ and suspected COVID-19 cases $(n=29)$ were reported a week earlier than the peak of confirmed cases during 3/16-3/22. Total number of confirmed, presumed, and suspected COVID-19 cases started to drop after the week of $3 / 23-3 / 29$. The bottom graph shows the number of programs enforcing mask policy by week. Most programs started to enforce universal mask policy during the week of $3 / 23-3 / 29$.

COVID-19 in NYC. Indeed, capturing the experience as it happens avoids recall bias after the fact. It is our hope that this insight may allow locations not yet as substantially affected by COVID-19 to better anticipate the needs of resident physicians, who are truly at the front lines of an unprecedented challenge.

\section{Methods}

Recruitment of program directors. Recruitment of residency program directors throughout the greater NYC area was performed through circulation of electronic mail message sent by one investigator at Columbia University Irving Medical Center, with responses received 
from April 3-12, 2020. Identification of programs, respective program directors, and contact electronic mail addresses were retrieved from either previous correspondence or publicly available search tools with ACGME via hyperlink (https://apps.acgme.org/ads/Public/ Programs/Search). The survey was first distributed to 12 ophthalmology residency program directors in the greater NYC area, who expanded distribution to 188 additional non-ophthalmology training programs within their own institutions. As a second method, 303 programs identified separately in the ACGME database by 2 authors of the present study were also contacted electronically. Ultimately, at least 1 contact attempt was made at every known residency training program in the greater NYC area (approximately 340 total), as our 2 approaches may have overlapped. Repeat contact for increased yield was not made because (a) initial feedback from designated institutional officials at some centers included concern for the potentially stressful nature of the survey despite institutional review board (IRB) approv$\mathrm{al}$, and (b) prolonging data collection time may have confounded results by including responses obtained outside of the pandemic exponential phase, introducing an element of recall bias.

Survey of resident physician experience. An anonymous survey (Supplemental Material) eliciting deidentified information was included in a circulated electronic mail message by hyperlink with SurveyMonkey cloud-based software (SurveyMonkey). More than one survey completion by the same user was prohibited, both by request within the recruitment electronic message and based on internet protocol address.

Diagnosis or suspicion of COVID-19 among residents was elicited in our survey based on clinical presentation with symptoms including: sore throat, cough, fever, shortness of breath, chest pain, myalgia, malaise, conjunctivitis, anosmia, or gastrointestinal symptoms. Survey questions pertained to 3 distinct groups among resident doctors (a) confirmed, defined as resident physicians with COVID-19 symptoms and positive test results; (b) presumed, defined as resident physicians with COVID-19 symptoms without test results; and (c) suspected, defined as resident physicians with COVID-19 symptoms and negative test results. Suspected cases were tallied in our analysis due to the relatively high false-negative rate of RT-PCR testing for active infection by this virus $(15,16)$ as well as high pretest probability for COVID-19 in the context of suggestive symptoms, due to health care worker status and NYC location.

Inclusion and exclusion of responses. Responses were reviewed for inclusion based on specific training program. Fellowship programs were excluded from the analysis. Because certain specialties have programs that exist as a residency-fellowship continuum, these training programs with ACGME accreditation were included. We did not distinguish between these integrated programs and residency-only programs. All programs included were ACGME accredited, with the exception of oral maxillofacial surgery (OMFS), which was included as many OMFS programs offer clinical experience through ACGMEaccredited rotations such as general surgery, ultimately leading to medical licensure with or without a medical degree, in addition to preexisting dental licensure. Programs were included if they were within or immediately adjacent to NYC. All queried programs but one were centralized within 30 miles of Central Park in Manhattan, verified by Google Maps with hyperlink: https://www.google.com/maps (Google Inc.) for distance calculations, which used mailing addresses from primary affiliations for each recipient of the survey.

Statistics. Proportions are reported as percentages with 95\% confidence interval calculated using the Clopper-Pearson approach. Specialties with representation by 100 or more residents were selected for further between-specialty analyses. Because the number of COVID-19-positive residents by individual programs were count outcomes and nonnormally distributed, Poisson regression and negative binomial regression were fitted to determine whether specialty, program size, or date of survey response affected the number of residents with positive COVID-19 tests. Likelihood ratio testing was used to determine the appropriateness between Poisson regression and negative binomial regression. Fisher's exact test was used to assess the overall effect of specialties on the proportion of residents with confirmed COVID-19. Pearson's $\chi^{2}$ test was used to compare infection rate and redeployment rate among departments. Correction for multiple comparisons was made with Bonferroni's procedures. Statistical analyses were performed in the R programming language (version.1.2.5042). Type 1 error was defined at the $5 \%$ level for hypothesis testing with 2-tailed probabilities.

Study approval. The need for subject consent was waived due to minimal risk, anonymous nature, and lack of sensitive information in the study design as per Columbia University IRB expedited exemption protocol IRB-AAAS9946. All procedures were reviewed and in accordance with the tenets of the Declaration of Helsinki.

\section{Author contributions}

MPB, AHA, JDH, and RWSC conceived the project. MPB, JS, AHA, LRDG, JDH, CGM, ACP, and RWSC designed the survey. MPB, JS, AHA, RWSC, and the New York City Residency Program Directors COVID-19 Research Group collected the data. MPB, JS, AHA, LRDG, JDH, SXX, CGM, ACP, and RWSC interpreted the data. MPB, JS, and RWSC prepared the figures. MPB, JS, AHA, LRDG, JDH, SXX, CGM, ACP, and RWSC prepared the manuscript.

\section{Acknowledgments}

We thank Julia A. Kucherich for contributing to survey design and discussion references. See Supplemental Acknowledgments for the New York City Residency Program Directors COVID-19 Research Group details. This work was supported by National Eye Institute Core Grant P3OEY019007 (AHA, MPB, RWSC, CGM, LRDG, JDH), a Research to Prevent Blindness Unrestricted Grant, the Parker Family Chair (ACP and JS), and the University of Pennsylvania (SXX).

Address correspondence to: Royce W.S. Chen, Columbia University Irving Medical Center, New York-Presbyterian Hospital, 635 West 165th Street, New York, New York 10032, USA. Phone: 212.305.9535; Email: rc2631@cumc.columbia.edu.
1. WHO. Novel Coronavirus (2019-nCoV): Situation Report-11. https://www.who.int/docs/ default-source/coronaviruse/situation-reports/20200131-sitrep-11-ncov.pdf?sfvrsn=- de7c0f7 4. Updated January 31, 2020. Accessed June 17, 2020.

2. Chen $\mathrm{N}$, et al. Epidemiological and clinical characteristics of 99 cases of 2019 novel coronavirus pneumonia in Wuhan, China: a descriptive study. Lancet. 2020;395(10223):507-513.

3. Li Q, et al. Early transmission dynamics in Wuhan, China, of novel coronavirus-infected pneumonia. 
NEngl JMed. 2020;382(13):1199-1207.

4. Live Science. Coronavirus in the US: Latest COVID-19 news and case counts. https://www. livescience.com/coronavirus-updates-united-states.html. Updated June 15, 2020. Accessed June 17, 2020.

5. West MG. First case of coronavirus confirmed in New York State. Wall Street Journal. March 1, 2020. https://www.wsj.com/articles/firstcase-of-coronavirus-confirmed-in-new-yorkstate-11583111692. Accessed June 17, 2020.

6. NYC Health. COVID-19: Data: Daily Counts. https://www1.nyc.gov/site/doh/covid/covid19-data.page. Updated June 17, 2020. Accessed June 17, 2020.

7. Wang X, Zhang X, He J. Challenges to the system of reserve medical supplies for public health emergencies: reflections on the outbreak of the severe acute respiratory syndrome coronavirus 2 (SARS-CoV-2) epidemic in China. Biosci Trends. 2020;14(1):3-8.

8. Buckley C. Chinese doctor, silenced after warning of outbreak, dies from coronavirus. The New York Times. February 6, 2020.https://nyti. ms/375KzwH. Accessed June 17, 2020.

9. Paulo AC, Correia-Neves M, Domingos T, Murta AG, Pedrosa J. Influenza infectious dose may explain the high mortality of the second and third wave of 1918-1919 influenza pandemic. PLoS ONE. 2010;5(7):e11655.

10. Lemon J. Hospital in Brooklyn looks like a "war zone", emergency room doctor says: "we're fighting for our own lives too." Newsweek. March 30, 2020. https://www.newsweek.com/hospitalbrooklyn-looks-like-war-zone-emergency-roomdoctor-says-were-fighting-our-own-1495007. Accessed June 17, 2020.

11. Association of American Medical Colleges. Report on Residents: Table B3: Number of Active Residents, by Type of Medical School, GME Specialty, and Sex: 2017-18 Active Residents. https://www.aamc.org/data-reports/ students-residents/interactive-data/table-b3number-active-residents-type-medical-schoolgme-specialty-and-sex. Updated August 27, 2018. Accessed June 17, 2020.

12. Accreditation Council for Graduate Medical Education. About Us. https://www.acgme.org/ About-Us/Overview. Accessed June 17, 2020.

13. Vargo E, et al. Cleveland Clinic Akron General Urology Residency Program's COVID-19 Experience. Urology. 2020;140:1-3.

14. Stoj VJ, Grant-Kels JM. Dermatology residents and the care of patients with coronavirus dis- ease 2019 (COVID-19). J Am Acad Dermatol. 2020;82(6):1572-1573.

15. Canelli R, Connor CW, Gonzalez M, Nozari A, Ortega R. Barrier enclosure during endotracheal intubation. $N$ Engl J Med. 2020;382(20):1957-1958.

16. Yu AY, Tu R, Shao X, et al. A comprehensive Chinese experience against SARS-CoV-2 in ophthalmology [published online April 7, 2020]. Eye Vis (Lond). https://doi.org/10.1186/s40662020-00187-2.

17. Wu P, et al. Characteristics of ocular findings of patients with coronavirus disease 2019 (COVID19) in Hubei Province, China. JAMA Ophthalmol. 2020;138(5):575-578.

18. Wang $\mathrm{W}$, et al. Detection of SARS-CoV-2 in different types of clinical specimens [published online March 11, 2020]. JAMA. 2020;323(18):1843-1844.

19. Xie C, et al. Comparison of different samples for 2019 novel coronavirus detection by nucleic acid amplification tests. Int J Infect Dis. 2020;93:264-267.

20. Bendavid E, et al. COVID-19 antibody seroprevalence in Santa Clara County, California [preprint]. https://doi:10.1101/2020.04.14.2006246 3. Posted on medRxiv April 30, 2020. 